\title{
TIME-PERIODIC CONVECTIVE PATTERNS IN A HORIZONTAL POROUS LAYER WITH THROUGH-FLOW
}

\author{
BY \\ FRANÇOISE DUFOUR (Institut de Mécanique des Fluides de Toulouse, UMR CNRS 5502 \\ IMFT/UPS/INP, Université Paul Sabatier, 118 route de Narbonne, 31062 Toulouse cedex, France) \\ AND \\ MARIE-CHRISTINE NÉEL (Institut de Mécanique des Fluides de Toulouse, UMR CNRS 5502 \\ IMFT/UPS/INP, Université Paul Sabatier, 118 route de Narbonne, 31062 Toulouse cedex, France)
}

\begin{abstract}
We investigate the time-periodic convective patterns which set on in an infinite porous layer saturated by a fluid, under the influence of a vertical temperature gradient, superimposed to a horizontal seeping through-flow. When the seepage velocity keeps moderate, it obeys the Darcy law, while the heat equation rules the evolution of the temperature. The transition towards convection is governed by the filtration Rayleigh number, and the above-mentioned system of partial differential equations has Galilean invariance: travelling waves, stationary with respect to a moving frame, solve this problem near the threshold. This follows directly from the study of natural convection. With the help of the center manifold theory, we show that other kinds of time-periodic two-dimensional structures exist: upstream to a fixed zone, their amplitude vanishes. Downstream, they resemble the travelling waves. Numerical simulation of the governing equations reproduces elements of both sets of convective structures, indexed by definite values of the time-frequency.
\end{abstract}

Introduction. A forced horizontal flow superimposed to a vertical temperature gradient can give rise to time-periodic convective structures. Many papers have been devoted to the case of a fluid layer, among which we note [4], [22], [23], and [25]. The experimental studies of [8] and [17] have treated the case of a horizontal porous layer, heated from below. They have put into evidence two qualitatively different regimes, beyond the convective onset; time-periodic patterns are observed at moderate values of the flowrate.

Received February 10, 1998.

2000 Mathematics Subject Classification. Primary 35K55, 35B10; Secondary 35B32.

Key words and phrases. Nonlinear equations of parabolic type, periodic solutions, bifurcation.

Current address: Département de Mécanique, Université Paul Sabatier, UFR MIG, bâtiment 1R2, 118 route de Narbonne, 31062 Toulouse cedex, France.

E-mail address: fd3@techprt.co.uk

Current address: Laboratoire d'Hydrodynamique Complexe, Université d'Avignon, 33 rue Pasteur, 84000 Avignon, France.

E-mail address: marie-christine.neel@univ-avignon.fr 
Larger values of the cross-flow give time-independent structures, in the form of rolls with axes parallel to the main flow. These results correspond to what is observed in fluid layers, when the Reynolds number is varied.

According to several authors the Darcy law is a not too bad model when the seepage velocity is moderate, in porous media with low permeability: the numerical works of [11] and [16], devoted to mixed and natural convection, have given the condition that the Darcy number (proportional to the permeability) be less than $10^{-3}$, respectively $10^{-4}$. Most experimental data of [8] correspond to this situation. Our final aim is to explain the various aspects of the transition towards convection in a porous layer, where a nonzero through-flow is imposed. Indeed, the Darcian or non-Darcian behaviour of the filtration speed influences the bifurcation: this motivates an accurate study of the different models. Here we begin with the Darcy law: it leads to a problem which can be worked out with mathematical tools; however, other models are the subject of forthcoming works. We focus on two-dimensional flows: indeed, the experiments of [8] and [17] have shown that in a rectangular duct heated from below the convective patterns are of this form, provided the seeping flowrate keeps moderate.

We study the filtration speed and the temperature in an infinite horizontal porous layer saturated by a fluid, when the through-flow is nonzero. On the bottom we impose a temperature $T_{0}^{*}$, while on the top it is $T_{1}^{*}<T_{0}^{*}$. We suppose that the seeping velocity is moderate and obeys the Darcy law. When $T_{0}^{*}-T_{1}^{*}$ is small, a linear temperature profile is observed inside the layer, and the filtration speed is uniform. This conductive configuration destabilizes when $T_{0}^{*}-T_{1}^{*}$ becomes larger than a critical value, as in the Rayleigh-Bénard problem. The nonzero value of the flowrate results in a Hopf bifurcation: we present an analytical description of all the time-periodic patterns that solve the Darcy law and the heat equation above the convective threshold. We will immediately see that this system admits solutions that are stationary with respect to a moving frame: among them travelling waves are found. Then, using the center manifold theory, we will show that the system also admits other solutions in the form of rolls with modulated amplitude. Analytical approximations to the convective patterns near the threshold will result from this study: they will be compared to a direct numerical simulation of the original system of partial differential equations.

After setting the physical problem and the mathematical formulation, we list the different families of convective structures, which are stationary with respect to a moving frame. Then we detail the arguments that ensure that the time-periodic small solutions are linked to an ordinary differential equation. From this we deduce that two families of time-periodic convective patterns solve the problem just above the convective threshold. One set is made of quasi-stationary travelling waves. Front-like patterns, which are not stationary with respect to any uniformly moving frame, are described with more details. This approach gives no information about stability: a numerical simulation allows us to observe both time-periodic structures.

1. Formulation of the physical problem. In a long porous pipe with rectangular cross section, a suitable vertical temperature gradient renders the conductive state unstable. We study the case when a non-vanishing horizontal through-flow is imposed. 
1.1. Physical Problem. Consider an isotropic homogeneous porous layer saturated by a fluid, with uniform thickness $H^{*}$, and of infinite extent in the horizontal $x$ direction. Let the temperature be $T_{0}^{*}$ on the bottom and $T_{1}^{*}<T_{0}^{*}$ on the top boundary. We choose $H^{*}$, $T_{0}^{*}-T_{1}^{*}, \frac{\lambda^{*}}{H^{*}(\rho c)_{f}^{*}}$ and $\frac{H^{* 2}(\rho c)^{*}}{\lambda^{*}}$ as references for length, temperature difference, seepage velocity and time. Here $(\rho c)_{f}^{*}, \lambda^{*}$ and $(\rho c)^{*}$ are the heat capacity per volume of the fluid, the thermal conductivity and the equivalent heat capacity per volume of the medium.

When the filtration speed is not too large it satisfies the Darcy law, according to [2], [11] and [16], while the temperature $\mathrm{T}$ is governed by the heat equation. Under the Boussinesq assumption, we arrive at the following system in its non-dimensional form:

$$
\begin{gathered}
\vec{V}=-\vec{\nabla} p+\operatorname{Ra} T \vec{e}_{z}, \\
\frac{\partial T}{\partial t}=-\vec{V} \cdot \vec{\nabla} T+\Delta T, \\
\nabla \cdot \vec{V}=0,
\end{gathered}
$$

where $p, \vec{V}, \vec{e}_{z}$ are the pressure, the filtration velocity field and the vertical unit vector, pointing upwards, respectively. The filtration Rayleigh number is $\mathrm{Ra}=\mathcal{R}^{2}=$ $\frac{K^{*} g^{*} \alpha^{*} H^{*}\left(T_{0}^{*}-T_{1}^{*}\right)(\rho c)_{f}^{*}}{\lambda^{*} \nu^{*}}$, with $K^{*}, g^{*}, \alpha^{*}$ and $\nu^{*}$ being the permeability of the medium, the gravitational acceleration, the thermal expansion coefficient and the kinematic viscosity of the fluid, respectively. The boundary conditions are $\vec{V} \cdot \vec{e}_{z}=0$ and $T=1$ at $z=0$, $\vec{V} \cdot \vec{e}_{z}=0$ and $T=0$ at $z=1$ and the horizontal flowrate is $\int_{0}^{1} \vec{V} \cdot \vec{e}_{x} d z=C$.

The conductive state $(T, \vec{V})=\left(1-z, C \vec{e}_{x}\right)$ satisfies (1.1)-(1.3) for all values of $\mathcal{R}$ and $C, \vec{e}_{x}$ being the horizontal unit vector pointing to the right. When $\mathcal{R}$ is larger than a critical value $\mathcal{R}_{c}$, this basic state becomes unstable.

1.2. Convective Patterns. The eigenmodes analysis of [3] shows that, in a porous layer with narrow rectangular cross section, planar eigenmodes destabilize the conductive state. Then, two-dimensional structures only are expected just above the critical value $\mathcal{R}_{c}=2 \pi$, which is equal to the $C=0$ case of natural convection. Moreover, the experiments of [8] and [17] produced planar flows only, for moderate values of $C$ inside ducts with large aspect ratios. For these reasons, we focus on the two-dimensional solutions of (1.1)-(1.3). Their evolution is governed by

$$
\begin{gathered}
\Delta \psi=\mathcal{R} \frac{\partial \theta}{\partial x} \\
\frac{\partial \theta}{\partial t}=\Delta \theta-C \frac{\partial \theta}{\partial x}+\mathcal{R} \frac{\partial \psi}{\partial x}+\frac{\partial \psi}{\partial z} \cdot \frac{\partial \theta}{\partial x}-\frac{\partial \psi}{\partial x} \cdot \frac{\partial \theta}{\partial z}
\end{gathered}
$$

and the boundary conditions are

$$
\theta=\psi=0 \quad \text { for } z=0 \text { or } z=1
$$

where $T=1-z+\theta / \mathcal{R}$, with $\psi$ being a stream function of $\vec{V}-C \vec{e}_{x}$.

The above-mentioned linear stability analysis shows that a Hopf bifurcation, with eigenfrequency $\pi C$ occurs when $\mathcal{R}=\mathcal{R}_{c}$ : time-periodic solutions are expected when $\mathcal{R}$ is slighty above $\mathcal{R}_{c}$. Some of them correspond to flows, which are quasi-stationary. We will see later that (1.4)-(1.6) also admits other solutions. 


\subsection{Quasi-stationary Convective Patterns.}

Since (1.1)-(1.3) is invariant under the mapping

$$
\mathcal{G}:(\vec{V}, p, T)(x, z, t) \mapsto\left(\vec{V}(x-C t, z, t)+C \vec{e}_{x}, p(x-C t, z, t)-C x, T(x-C t, z, t)\right),
$$

it admits solutions that are stationary in a frame, moving at speed $C \vec{e}_{x}$. With $X=x-C t$, the solutions to (1.1)-(1.3) of the form

$$
(\vec{V}, T, p)=\left(\left(C-\partial_{z} \psi(X, z)\right) \vec{e}_{x}+\partial_{x} \psi \vec{e}_{z}, 1-z+\mathcal{R}^{-1} \theta(X, z), p(X, z)-C x\right)
$$

are such that $\psi(X, z)$ and $\theta(X, z)$ satisfy the time-independent version of (1.4)-(1.6) with $C=0$ for $(X, z) \in \mathbb{R} \mathbf{x}[0,1]$. General results of [14] were applied by [21] to this problem. Thus, for small values of $\mathcal{R}-\mathcal{R}_{c}=\mu$ and all variations of $C$, the small real-valued solutions to (1.1) $-(1.3)$ which are of the form (1.7) are such that

$$
(\theta, \psi)^{\dagger}=A_{1}(X) W_{1}(z)+A_{2}(X) W_{2}(z)+\text { c.c. }+\Lambda\left(A_{1}, A_{2}, \overline{A_{1}}, \overline{A_{2}}, \mu\right)
$$

where $\Lambda$ is regular with respect to its arguments while the $W_{i}$ are linked to the eigenvectors of some linear operator. The $A_{i}$ solve the 1:1 resonant normal form equation:

$$
\begin{gathered}
\frac{d A_{1}}{d x}=i \pi A_{1}+\left(1+2 \pi^{2}\right) A_{2}+i A_{1} \mathcal{P}_{1}\left(\left|A_{1}\right|^{2}, \frac{i}{2}\left(A_{1} \overline{A_{2}}-\overline{A_{1}} A_{2}\right), \mu\right)+f_{1} \\
\frac{d A_{2}}{d x}=i \pi A_{2}+i A_{2} \mathcal{P}_{1}+A_{1} \mathcal{P}_{2}\left(\left|A_{1}\right|^{2}, \frac{i}{2}\left(A_{1} \overline{A_{2}}-\overline{A_{1}} A_{2}\right), \mu\right)+f_{2}
\end{gathered}
$$

Here the $\mathcal{P}_{i}$ are real polynomials of arbitrary degree $q$ and the $f_{i}\left(A_{1}, A_{2}, \overline{A_{1}}, \overline{A_{2}}, \mu\right)$ are smooth, of order $2 q+1$, with respect to $A_{i}$ and $\bar{A}_{i}$, according to [14]. Since the problem with $C=0$ is reversible, the normal form (1.8)-(1.9) admits periodic and quasi-periodic (with respect to $X$ ) solutions following [14] and [12]. The periodic ones form a family $P\left(\mu, k, X_{0}\right)$, where $X_{0}$ stands for arbitrary translations along $X$, while the wave-number $k$ is in a neighbourhood of $\pi$. Their amplitude is proportional to $\sqrt{\pi \mu-(k-\pi)^{2}}$. Since the coefficient of $\left|A_{1}\right|^{2}$ in $\mathcal{P}_{2}$ has the sign of the coefficient of $\mu$ (see [21]), the normal form (1.8)-(1.9) also admits solutions, denoted by $H$, that are homoclinic to periodic ones at infinity following [15]. Therefore (1.1)-(1.3) has solutions that are periodic with respect to $X$ and stationary in the frame moving at speed $C \vec{e}_{x}$. From the laboratory frame, they are seen as being travelling waves with time-frequency $k C=\omega$.

The quasi-periodic solutions of (1.8)-(1.9) resemble periodic structures with approximate wavelength 2 ; their amplitude is modulated at larger scale $O\left(\mu^{-1 / 2}\right)$. From the laboratory frame, the corresponding structures are seen as being quasi-periodic with respect to $x$ and $t$. In the moving frame, the convective structures associated to elements of the family $H$ resemble the $X$ periodic ones, except in a finite zone where the amplitude is weaker, but the defect is swept away downstream. Imagine an observer unable to appreciate what happens outside a finite portion of the porous layer: at large times, he would see no difference between travelling waves and the structures corresponding to the family $H$. For this reason, we focus our attention on time-periodic patterns that may not be quasi-stationary. 
2. Time-periodic solutions of (1.4)-(1.6). System (1.4)-(1.6), for $\mathcal{R}$ near $\mathcal{R}_{c}$, enters the general framework of [13] where a similar problem without the reflectional symmetry was studied. Considering time-periodic solutions of (1.4)-(1.6) with frequency $\omega$ introduces this arbitrary parameter into the equations, not in the definition of the space where solutions are sought. We will show that the obtained mathematical problem has solutions, associated to an ordinary differential equation. Among them, we will recover the travelling rolls that correspond to the family $P$. We will also find other convective patterns.

2.1. Reformulation of (1.4)-(1.6). System (1.4)-(1.6) is equivalent to

$$
\partial_{x} \mathcal{U}=L_{\mathcal{R}, C, \omega}(\mathcal{U})+N(\mathcal{U}, \mathcal{U})
$$

with $\mathcal{U}=(\theta, \Theta, \psi, \Psi,)^{\dagger}, \tau=\omega t$ and

$$
\begin{gathered}
L_{\mathcal{R}, C, \omega} \mathcal{U}=\left(\Theta,-\partial_{z^{2}}^{2} \theta+C \Theta-\mathcal{R} \Psi+\omega \partial_{\tau} \theta, \Psi,-\partial_{z^{2}}^{2} \psi+\mathcal{R} \Theta\right)^{\dagger}, \\
N\left(\mathcal{U}_{1}, \mathcal{U}_{2}\right)=\left(0, \Psi_{1} . \partial_{z} \theta_{2}-\Theta_{1} . \partial_{z} \psi_{2}, 0,0\right)^{\dagger} .
\end{gathered}
$$

The superscript $\dagger$ denotes the transpose, and $\mathcal{U}_{j}=\left(\theta_{j}, \Theta_{j}, \psi_{j}, \Psi_{j}\right)^{\dagger}$. The solutions of (1.4)-(1.6), which are $2 \pi \omega^{-1}$-periodic with respect to $t$, correspond to solutions of $(2.1)$ with period $2 \pi$ w.r.t. $\tau$.

2.2. Functional Setting. The linear operator $L_{\mathcal{R}, C, \omega}$ is continuous from the Hilbert space

$$
\mathcal{D}=\left(\mathcal{H}_{0}^{0,2} \cap \mathcal{H}^{1,0}\right) \times\left(\mathcal{H}_{0}^{0,1} \cap \mathcal{H}^{1 / 2,0}\right) \times\left(\mathcal{H}^{1 / 2,2} \cap \mathcal{H}_{0}^{1 / 2,1}\right) \times \mathcal{H}_{0}^{1 / 2,1}
$$

to

$$
\mathcal{H}=\mathcal{H}_{0}^{0,1} \cap \mathcal{H}^{1 / 2,0} \times \mathcal{H}^{0,0} \times \mathcal{H}_{0}^{1 / 2.1} \times \mathcal{H}^{1 / 2.0} .
$$

Here $(\tau, x)$ belongs to $S^{1} \times[0,1]$, and with the notation of [13], $\mathcal{H}^{\sigma, r}=H^{\sigma}\left(S^{1}, H^{r}[0,1]\right)$ is endowed with the norm defined for every $u(\tau,)=.\sum_{n \in \mathbb{Z}} u_{n}(.) e^{i n \tau}$ by $\|u\|_{\sigma, r}^{2}=$ $\sum_{n \in \mathbb{Z}}\left(1+n^{2}\right)^{\sigma}\left\|u_{n}\right\|_{H^{r}[0,1]}^{2}$. For $r \geq 1$ we also use $\mathcal{H}_{0}^{\sigma, r}=H^{\sigma}\left(S^{1}, H^{r}[0,1] \cap H_{0}^{1}[0,1]\right)$ where (as usual) $H_{0}^{1}[0,1]=\left\{v \in H^{1}[0,1] / v(0)=v(1)=0\right\}$.

The smoothness of $N$ and spectral properties of $L_{\mathcal{R}, C, \omega}$ will be shown to imply that (2.1) admits a finite-dimensional center manifold, in view of a theorem of [19]. While the main ideas in the proof of these properties have been introduced in [13], it is not possible to use directly here the theorems of these authors. Indeed, the estimate (2.9) in Lemma 3 below is only valid for the resolvent of $L_{\mathcal{R}, C, \omega}$, restricted to a closed submanifold of $\mathcal{H}$, as we will see in Sec. 2.4. For this reason, we have to adapt their proof.

\subsection{Regularity of $N$. We have the lemma:}

Lemma 1. The mapping $\mathcal{U} \longmapsto N(\mathcal{U}, \mathcal{U})$ is $\mathcal{C}^{k}(\mathcal{D}, \mathcal{H})$ for every natural number $k$.

Proof. It is enough to show that the quadratic mapping $N$ is continuous separately with respect to each variable. This is a consequence of the continuity of the product of $u \in \mathcal{H}^{0,1} \cap \mathcal{H}^{1,-1}$ or $\mathcal{H}_{0}^{0,1} \cap \mathcal{H}^{1 / 2,0}$ by $v \in \mathcal{H}^{1 / 2,1}$ as a mapping taking its values in $\mathcal{H}^{0,0}$.

Consider $u \in \mathcal{H}^{0,1} \cap \mathcal{H}^{1,-1}$ first. Let us take $\lambda$ in ]0,1/2[. With $\gamma=1-2 \lambda$ and according to the notation of $[18],\left[H_{0}^{1}[0,1], H^{-1}[0,1]\right]_{\lambda}=H^{\gamma}[0,1]$. Expand $u=\sum_{n \in \mathbb{Z}} u_{n} e^{i n \tau}$ in 
Fourier series with respect to $\tau:\|u\|_{\mathcal{H}^{0.1} \cap \mathcal{H}^{1,-1}}^{2}=\sum_{n \in \mathbb{Z}}\left(\left(1+n^{2}\right)\left\|u_{n}\right\|_{H^{-1}[0,1]}^{2}+\right.$ $\left.\left\|u_{n}\right\|_{L^{2}[0.1]}^{2}\right)$. We have

$$
\left\|u_{n}\right\|_{H^{1}[0.1]}^{1-\lambda}\left\|u_{n}\right\|_{H^{-1}[0.1]}^{\lambda}\left(1+n^{2} / 2\right)^{\lambda / 2} \leq \sqrt{\left\|u_{n}\right\|_{H^{1}[0.1]}^{2}+\left(1+n^{2}\right)\left\|u_{n}\right\|_{H^{-1}[0.1]}^{2}}
$$

and therefore $\|u\|_{\mathcal{H}^{\lambda, \gamma}} \leq\|u\|_{\mathcal{H}^{0,1} \cap \mathcal{H}^{1,-1}}$.

In view of Sobolev inclusions [1], $\mathcal{H}^{\lambda, \gamma} \hookrightarrow L^{p}\left(S^{1}, L^{q}[0,1]\right)$ for $\frac{1}{p}>\frac{1}{2}-\lambda$ and $\frac{1}{q}>\frac{1}{2}-\gamma$. Moreover, for $p^{\prime}>2, \mathcal{H}^{\frac{1}{2} \cdot 1} \hookrightarrow L^{p^{\prime}}\left(S^{1}, \mathcal{C}^{0}[0,1]\right)$ and there exist $p, p^{\prime}, q$ such that $\frac{1}{p}>\frac{1}{2}-\lambda$, $\frac{1}{q}>\frac{1}{2}-\gamma, p^{\prime}>2, \frac{1}{p}+\frac{1}{p^{\prime}} \leq \frac{1}{2}$. With $v \in \mathcal{H}^{1 / 2.1}$ we also have

$$
\begin{aligned}
\|u v\|_{L^{2}\left(S^{1} \cdot L^{2}[0.1]\right)} & \leq \mathcal{K}\|u\|_{L^{p}\left(S^{1} \cdot L^{q}[0,1]\right)}\|v\|_{L^{p^{\prime}\left(S^{1}, \mathcal{C}^{0}[0.1]\right)}} \\
& \leq \mathcal{K}_{1}\|u\|_{\mathcal{H}^{0,1} \cap \mathcal{H}^{1,-1}}\|v\|_{\mathcal{H}^{1 / 2.1}} .
\end{aligned}
$$

Similarly with $u \in \mathcal{H}_{0}^{(0.1} \cap \mathcal{H}^{1 / 2.0}$, since $H^{1 / 2}[0,2 \pi] \hookrightarrow L^{p}[0,2 \pi]$ for $p>2$, and recalling that $\|v\|_{L^{q}\left(S^{1} \cdot \mathcal{C}^{0}([0.1])\right.} \leq \mathcal{K}_{2}\|v\|_{\mathcal{H}^{1 / 2.1}}$ for $q>2$, we have

$$
\|u v\|_{L^{2}\left(S^{1} \cdot L^{2}[0,1]\right)} \leq \mathcal{K}_{3} \quad\|u\|_{\mathcal{H}^{1 / 2,0} \cap \mathcal{H}^{0.1}}\|v\|_{\mathcal{H}^{1 / 2,1}} .
$$

Set $u=\partial_{z} \theta$ and $v=\psi$. Then $u=\Theta$ and $v=\partial_{z} \psi$. The quadratic mapping $\left(\mathcal{U}_{1}, \mathcal{U}_{2}\right) \longmapsto N\left(\mathcal{U}_{1}, \mathcal{U}_{2}\right)$ is continuous from $\mathcal{D}$ towards $\mathcal{H}$, hence Lemma 1 .

We will see that the spectrum of $L_{\mathcal{R} . C . \omega}$ is discrete in a neighbourhood of the imaginary axis, where the eigenvalues have finite multiplicity, and state estimates for the resolvent of $L_{\mathcal{R} . C . \omega}$.

2.4. Spectral Properties of $L_{\mathcal{R}, C . \omega}$. Let us show that the resolvent $\left(L_{\mathcal{R} . C, \omega}-\lambda \mathrm{Id}\right)^{-1}$ exists for larg€ $\lambda$ in a neighbourhood of the imaginary axis, with bounds for $\left(L_{\mathcal{R} . C . \omega}-\right.$ $\lambda \mathrm{Id})^{-1}$ and $\left(L_{\mathcal{R} . C . \omega}-\lambda \mathrm{Id}\right)^{-2}$ in restriction to the closed submanifold $\{0\} \times \mathcal{H}^{0.0} \mathbf{x}\{0\}^{2}$ of $\mathcal{H}$. We also need $L_{\mathcal{R} \text {.C. } \omega}$ to have a finite number of eigenvalues in a neighbourhood of the imaginary axis.

LEMma 2. For large real $s$ and $(f, g)$ in $\mathcal{H}^{(0.0} \times \mathcal{H}^{1 / 2.0}$, the problem

$$
\left(-\partial_{z^{2}}^{2} \theta+s^{2} \theta+\omega \partial_{\tau} \theta,-\partial_{z^{2}}^{2} \psi+s^{2} \psi\right)=(f, g)
$$

has one solution $R_{s}(f, g)$ in $\left(\mathcal{H}^{0.2} \cap \mathcal{H}_{0}^{1.0}\right) \times\left(\mathcal{H}^{1 / 2.2} \cap \mathcal{H}_{0}^{1 / 2.1}\right)$ satisfying

$$
\|(\theta, \psi)\|_{\left(\mathcal{H}^{0.2} \cap \mathcal{H}_{0}^{1,0}\right) \times \mathcal{H}^{1 / 2.2}} \leq K_{1}(\omega)\|(f, g)\|_{\mathcal{H}^{0.0} \times \mathcal{H}^{1 / 2.0}}
$$

and

$$
\begin{gathered}
\|(\theta, \psi)\|_{\tilde{\mathcal{H}}_{i}} \equiv|s|\|\theta\|_{\mathcal{H}^{0.0}}+\|\theta\|_{\mathcal{H}^{0.1}}+\|\theta\|_{\mathcal{H}^{1 / 2.0}}+|s|\|\psi\|_{\mathcal{H}^{1 / 2.0}}+\|\psi\|_{\mathcal{H}^{1 / 2,1}} \\
\leq K_{1}(\omega)|s|^{-1}\left(\|f\|_{\mathcal{H}^{0.0}}+\|g\|_{\mathcal{H}^{1 / 2.0}}\right)
\end{gathered}
$$

A consequence of this lemma, which itself is proved in subsection 5.1 , is that

$$
\left\|R_{s}(f, g)\right\|_{\dot{\mathcal{H}}_{i}} \leq K_{1}(\omega)|s|^{-1}\|(0, f, 0, g)\|_{\mathcal{H}} .
$$

REMARK 2.4.1. Provided $f$ and $g$ belong to $\mathcal{H}^{1 / 2.1}$, we have

$$
\left\|R_{s}(f, g)\right\|_{\tilde{\mathcal{H}}_{*}} \leq K_{1}(\omega) s^{-2}\|(f, g)\|_{\tilde{\mathcal{H}}_{s}} .
$$

From this, in subsection 5.2 we deduce: 
Lemma 3. For every $(h, f, k, g)$ in $\mathcal{H}$, there exist positive real numbers $s_{0}(\mathcal{R}, C, \omega)$ and $\alpha_{0}(\mathcal{R}, C, \omega)$ such that for $|s| \geq s_{0}$ and $|\alpha| \leq \alpha_{0}$, the problem

$$
\left(L_{\mathcal{R} . C . \omega}-(i s+\alpha) \mathrm{Id}\right) U=(h, f, k, g)
$$

has a unique solution $U=(\theta,(i s+\alpha) \theta, \psi,(i s+\alpha) \psi)$ in $\mathcal{D}$. When $h=k=0$ it satisfies

$$
\|U\|_{\mathcal{H}} \leq\|(\theta, \psi)\|_{\tilde{\mathcal{H}}_{s}} \leq K^{\prime}(\mathcal{R}, C, \omega)|s|^{-1}\|(0, f, 0, g)\|_{\mathcal{H}} .
$$

REMARK 2.4.2. Estimate (2.9) is not valid for every $(h, f, k, g)$ in $\mathcal{H}$. To see this, for every real $s$ with $s>\mathcal{R}$, take a sequence $a_{n}$ of nonnegative real numbers, such that the corresponding series converges. With $q(n)=E\left(\sqrt{\frac{n \omega}{\pi^{2}}}\right)$ and $n_{1}=\max \left(s^{2} / \omega, c s / \omega\right)$, take $h=\sum_{n \geq n_{1}} e^{i n \tau} a_{n} \sin (q(n) \pi z)$, and solve $\left(L_{\mathcal{R} . C . \omega}-i s\right.$ Id $) U=(h, 0,0,0)$ in $\mathcal{D}$. The second component $\Theta$ of $U$ satisfies $6\|\Theta\|_{\mathcal{H}^{0.0}} \geq\|(h, 0,0,0)\|_{\mathcal{H}}$, hence the remark. For this reason, Lemma 4, proved in subsection 5.2, is necessary to ensure that the hypotheses of Theorem 1 of [19] are satisfied.

LEMma 4. For $|\operatorname{Re} \lambda| \leq \alpha_{0}(\mathcal{R}, C, \omega)$ and $|\operatorname{Im} \lambda| \geq s_{0}(\mathcal{R}, C, \omega)$, the problem

$$
\left(L_{\mathcal{R} . C . \omega}-\lambda \mathrm{Id}\right)^{2} U^{\prime}=(0, f, 0,0)
$$

has a unique solution $U^{\prime}=\left(\theta^{\prime}, \Theta^{\prime}, \psi^{\prime}, \Psi^{\prime}\right)$ in $\mathcal{D}$, with

$$
\left\|U^{\prime}\right\|_{\mathcal{H}} \leq K(\mathcal{R}, C, \omega)|\lambda|^{-2}\|(0, f, 0,0)\|_{\mathcal{H}}
$$

Now $\left\{\lambda \in \mathbb{C} /|\operatorname{Re} \lambda| \leq \alpha_{0},|\operatorname{Im} \lambda| \geq s_{0}\right\}$ is included in the resolvent set of $L_{\mathcal{R}, C . \omega}$. We still have to show that $\left(L_{\mathcal{R}, C, \omega}-\lambda \mathrm{Id}\right)^{-1}$ is well defined in

$$
\rho_{\alpha_{0}, s_{0}} \equiv\left\{\lambda \in \mathbb{C} /|\operatorname{Re} \lambda| \leq \alpha_{0},|\operatorname{Im} \lambda| \leq s_{0}\right\}
$$

with the exception of a finite number of values of $\lambda$. This is not obvious a priori: we do not know whether the spectrum of $L_{\mathcal{R}, C, \omega}$ is discrete since the embedding $\mathcal{D} \hookrightarrow \mathcal{H}$ is not compact. Indeed, there is no time derivative in the fourth component of $L_{\mathcal{R} . C . \omega}$, hence no temporal smoothing for $\psi$ in the resolvent. Nevertheless this part of the proof, which results in Lemma 5, follows exactly [13] and is based upon the fact that the singularities of $\left(L_{\mathcal{R} . C . \omega}-\lambda \mathrm{Id}\right)^{-1}$ concentrate at low frequencies. In subsection 5.3 we prove:

LEMMA 5. The resolvent $\left(L_{\mathcal{R}, C, \omega}-\lambda \mathrm{Id}\right)^{-1}$ exists in $\rho_{\alpha_{0}, s_{0}}$ except for a finite number of $\lambda$, which are eigenvalues of $L_{\mathcal{R} . C, \omega}$ with finite multiplicities. The corresponding eigenfunctions are finite sums of terms such as $e^{i n \tau} \mathcal{W}_{n}(z)$.

Lemmas 2 and 5 imply that the resolvent of $L_{\mathcal{R}, C \cdot \omega}$ exists in the strip $\{\lambda \in \mathbb{C} /|\operatorname{Re} \lambda| \leq$ $\left.\alpha_{0}\right\}$, with the exception of a finite set of eigenvalues with finite multiplicities.

3. Reduced ordinary differential equation for (2.1). This result, the estimates (2.9) and (2.11) and Lemma 1 imply that the small solutions of (2.1) are drawn on a finitedimensional center manifold and are associated with an ordinary differential equation. 
3.1. Center Manifold for (2.1). For real $\mathcal{R}_{0}$ and $\omega_{0}$, let

$$
E_{c}\left(\mathcal{R}_{0}, C, \omega_{0}\right)=\bigoplus_{s \in \mathbb{R}} \bigcup_{n \in \mathbb{N}} \operatorname{Ker}\left(L_{\mathcal{R}_{0}, C, \omega_{0}}-i s \mathrm{Id}\right)^{n}
$$

be the central eigenspace of $L_{\mathcal{R}_{0}, C, \omega_{0}}$; if this operator has no purely imaginary eigenvalues, $E_{c}=\{O\}$.

If $E_{c} \neq\{O\}$, let $\Gamma$ be a closed curve in $\left\{\lambda \in \mathbb{C} /|\operatorname{Re} \lambda| \leq \alpha_{0}\right\}$, surrounding all the purely imaginary eigenvalues of $L_{\mathcal{R}_{0}, C, \omega_{0}}$, and only them: $E_{c}$ is the range of the projection

$$
P_{\mathcal{R}_{0}, C, \omega_{0}}=\frac{1}{2 i \pi} \int_{\Gamma}\left(L_{\mathcal{R}_{0}, C . \omega_{0}}-\lambda \mathrm{Id}\right)^{-1} d \lambda .
$$

Let us call $\mathcal{T}_{\tau_{0}}$ the translation with amplitude $\tau_{0}$ in the direction of $\tau$. Lemmas 1-5 imply that the hypotheses of Theorem 1 of [19] are satisfied. Since also (2.1) is autonomous we have:

ThEOREM 1. For $\left(\mathcal{R}_{0}, \omega_{0}\right) \in \mathbb{R}^{2}$ there exist neighbourhoods $\mathcal{V}_{1}$ of $\left(\mathcal{R}_{0}, \omega_{0}\right)$ in $\mathbb{R}^{2}$ and $\mathcal{V}_{2}$ of $O$ in $\mathcal{D}$ such that

(i) for $(\mathcal{R}, \omega)$ in $\mathcal{V}_{1}$, the solutions of $(2.1)$, taking their values in $\mathcal{V}_{2}$, are of the form

$$
U_{c}+\hbar\left(\mathcal{R}, C, \omega, U_{c}\right)
$$

with $U_{c} \in E_{c}\left(\mathcal{R}_{0}, C, \omega_{0}\right), \hbar$ taking its values in the range of Id $-P_{\mathcal{R}_{0}, C, \omega_{0}}$, with

$$
\hbar\left(\mathcal{R}_{0}, C, \omega_{0}, O\right)=\frac{\partial \hbar}{\partial U_{c}}\left(\mathcal{R}_{0}, C, \omega_{0}, O\right)=0 .
$$

(ii) When $E_{c}\left(\mathcal{R}_{0}, C, \omega_{0}\right) \neq\{O\}$, set

$$
\mathcal{F}\left(\mathcal{R}, C, \omega, U_{c}\right) \equiv P_{\mathcal{R}_{0}, C, \omega_{0}}\left(L_{\mathcal{R}, C, \omega}\left(U_{c}+\hbar\left(\mathcal{R}, C, \omega, U_{c}\right)\right)+N\left(U_{c}+\hbar\left(\mathcal{R}, C, \omega, U_{c}\right), U_{c}+\hbar\right)\right):
$$

every small solution of

$$
\frac{d U_{c}}{d x}=\mathcal{F}\left(\mathcal{R}, C, \omega, U_{c}\right)
$$

yields through $U_{c}+\hbar\left(\mathcal{R}, C, \omega, U_{c}\right)$ a solution to $(2.1)$.

(iii) For every real $\tau_{0}, \mathcal{T}_{\tau_{0}} \mathcal{F}\left(\mathcal{R}, C, \omega, U_{c}\right)=\mathcal{F}\left(\mathcal{R}, C, \omega, \mathcal{T}_{\tau_{0}} U_{c}\right)$.

3.2. Dimension of $E_{c}$. The dimension of $E_{c}\left(\mathcal{R}_{0}, C, \omega_{0}\right)$ gives the number of degrees of freedom of (3.2). For $\mathcal{R}_{0} \leq \mathcal{R}_{c}=2 \pi, L_{\mathcal{R}_{0}, C, \omega_{0}}$ has no purely imaginary eigenvalues: (2.1) has no solutions near $O$; hence (1.4)-(1.6) has no time-periodic solutions near the conductive state.

For $\mathcal{R}_{0}=\mathcal{R}_{c}$ and $\omega_{0}=\omega_{c}=\pi C, L_{\mathcal{R}_{0}, C, \omega_{0}}$ admits two purely imaginary eigenvalues $\pm i \pi$, with eigenvectors $W$ and $\bar{W}$ such that

$$
W=e^{-i \tau} \sin \pi z(1, i \pi,-i, \pi)^{\dagger} .
$$

Other values of $\omega_{0}$ give no purely imaginary eigenvalues (with the exception of the $\omega_{c} / n$ which only are a reformulation of the same case).

When $\mathcal{R}_{0}=\mathcal{R}_{c}+\nu$ with small nonnegative $\nu, L_{\mathcal{R}_{0}, C \text {. } \omega_{0}}$ admits two purely imaginary eigenvalues $\pm i s$, which are either $\pm i\left(2 \pi+\nu+\sqrt{4 \pi \nu+\nu^{2}}\right) / 2$ or $\pm i\left(2 \pi+\nu-\sqrt{4 \pi \nu+\nu^{2}}\right) / 2$ provided $\omega_{0}$ is $C\left(2 \pi+\nu+\sqrt{4 \pi \nu+\nu^{2}}\right) / 2$ or $C\left(2 \pi+\nu-\sqrt{4 \pi \nu+\nu^{2}}\right) / 2$ : except when $C=0$, no $\omega_{0}$ can satisfy both conditions alltogether. The associated eigenvectors 
are $e^{-i \tau} \sin \pi z(1, i s,-i, s)^{\dagger}$ and the conjugate. To see that when $C \neq 0$ the eigenvalues $\pm i s$ of $L_{\mathcal{R}_{0}, C, \omega_{0}}$ are simple, one can notice that the equation $\left(L_{\mathcal{R}_{0}, C . \omega_{0}}-i s\right) X=$ $\sin (\pi z) e^{-i \tau}(F, G, H, J)^{\dagger}$ has solutions if and only if

$$
F \mathcal{R}_{0}\left(i s C-\pi^{2}\right)-\mathcal{R}_{0} i s G-i s\left(\mathcal{R}_{0}^{2}-\pi^{2}-s^{2}\right) H+\left(\pi^{2}+s^{2}\right) J=0 .
$$

To study (2.1) when $\mathcal{R}$ is just above $\mathcal{R}_{c}$, it is enough to consider $\mathcal{R}_{0}=\mathcal{R}_{c}$. When $C$ stays outside of a neighbourhood of zero the eigenvalues $\pm i \pi$ of $L_{\mathcal{R}_{c}}$.C. $\omega_{c}$ are simple: this is a consequence of the solvability condition

$$
F(i C-\pi)-i G-i \pi H+J=0
$$

for the equation $\left(L_{\mathcal{R}_{c}, C, \omega_{c}}-i \pi\right) X=\sin (\pi z) e^{-i \tau}(F, G, H, J)^{\dagger}: W$ and $\bar{W}$ are not in the range of $L_{\mathcal{R}_{c}, C, \omega_{c}} \pm i \pi$ Id when $C \neq 0$; hence $E_{c}\left(\mathcal{R}_{c}, C, \omega_{c}\right)$ is spanned by $W$ and the conjugate.

3.3. Symmetries of $\mathcal{F}$. When $\mu$ is small in $\mathcal{R}=\mathcal{R}_{c}+\mu$, with $\omega$ near $\omega_{c}$, the small real solutions to (2.1) are of the form

$$
A W+\overline{A W}+\Lambda\left(A, \bar{A}, \mu, \omega-\omega_{c}\right),
$$

where $\Lambda$ and its derivative vanish when $\left(A, \bar{A}, \mu, \omega-\omega_{c}\right)=O$. In view of point (iii) in the theorem, with $\mathcal{F}=\mathcal{F}_{1} W+\mathcal{F}_{2} \bar{W}$ we have

$$
\mathcal{F}_{j}\left(\mathcal{R}, C, \omega, A e^{-i \tau_{0}} W+\bar{A} e^{i \tau_{0}} \bar{W}\right)=e^{(-1)^{j} i \tau_{0}} \mathcal{F}_{j}(\mathcal{R}, C, \omega, A W+\overline{A W}) .
$$

Thus the complex-valued function $A$ of $x$ satisfies the ordinary differential equation

$$
\frac{d A}{d x}=A\left(i \pi+\alpha \mu+\beta\left(\omega-\omega_{c}\right)+\gamma|A|^{2}+\zeta\left(\omega-\omega_{c}\right)^{2}+\tilde{\mathcal{F}}\left(|A|^{2}, \mu, \omega-\omega_{c}\right)\right),
$$

where the order of magnitude of $\tilde{\mathcal{F}}$ is $\left(|A|^{2}+\mu\right)\left|\omega-\omega_{c}\right|+\left(|A|^{2}+\mu+\left|\omega-\omega_{c}\right|^{2}\right)^{2}$. Substituting (3.4) and (3.5) into (2.1) and identifying the coefficients of the $A^{p} \bar{A}^{q} \mu^{r}\left(\omega-\omega_{c}\right)^{\sigma}$, we obtain

$$
\left(L_{\mathcal{R}_{c}, C, \omega_{c}}-\lambda \mathrm{Id}\right) \Lambda_{p q}^{r \sigma}=Y_{p q}^{r \sigma}
$$

with $\lambda=0, i \pi$ or $2 i \pi, \ldots 2 i m \pi, m$ being an integer, and where the $Y_{p q}^{r \sigma}$ with $p+q+r+\sigma=$ $n$ depend on the $Y_{p^{\prime} q^{\prime}}^{r \prime \sigma^{\prime}}$ with $p^{\prime}+q^{\prime}+r \prime+\sigma \prime<n$. The $\Lambda_{p q}^{r \sigma}$ represent the coefficients in the Taylor expansion of $\Lambda$ around $O$ :

$$
\Lambda\left(A, \bar{A}, \mu, \omega-\omega_{c}\right)=\sum_{p+q+r+\sigma \geq 2} \Lambda_{p q}^{r \sigma} A^{p} \bar{A}^{q} \mu^{r}\left(\omega-\omega_{c}\right)^{\sigma} .
$$

The condition (3.3) yields $\alpha=\frac{2 \pi}{C}, \beta=\frac{i}{C}, \gamma=-\frac{\pi^{2}}{2 C}, \zeta=-2 C^{-3}$.

3.4. Bounded Solutions of (3.5). With $A(x)=r(x) e^{i \varphi(x)}$, and real $r$ and $\varphi,(3.5)$ is equivalent to

$$
\begin{gathered}
\frac{d r}{d x}=r M\left(r, \mu, \omega-\omega_{c}\right)=\frac{\pi}{C} r \cdot\left(2 \mu-\frac{\pi}{2} r^{2}-2 \frac{\left(\omega-\omega_{c}\right)^{2}}{\pi C^{2}}+g_{1}\left(r^{2}, \mu, \omega-\omega_{c}\right)\right), \\
\frac{d \varphi}{d x}=\pi+\frac{\omega-\omega_{c}}{C}+g_{2}\left(r^{2}, \mu, \omega-\omega_{c}\right)
\end{gathered}
$$

for small $\mu$ and $\omega$ close to $\omega_{c}=\pi C$, and where the $g_{j}$ are of the order of magnitude of

$$
O\left(\left(r^{2}+\left(\omega-\omega_{c}\right)^{2}+|\mu|\right)\left(\omega-\omega_{c}\right)\right)+O\left(r^{2}+O\left(r^{2}+\left(\omega-\omega_{c}\right)^{2}+|\mu|\right)^{2}\right) .
$$


Every trajectory of (3.6)-(3.7) gives rise to a family of solutions, up to arbitrary translations along $x$. On bounded trajectories, $r$ has to travel between successive roots of the right-hand side of (3.6) which are the stationary points of this equation. For small nonnegative $\mu$ the roots are 0 and $r_{*} \simeq 2 \sqrt{\mu / \pi-\left(\left(\omega-\omega_{c}\right) / \omega_{c}\right)^{2}}$. The other bounded trajectories of (3.6) correspond to functions $r_{x_{0}}$ of $x$ taking their values in $\left[0, r_{*}\right]$ and, according to [13], implicitly given by

$$
x=x_{0}+\int_{r_{*} / 2}^{r_{r_{0}}(x)}\left(\rho\left(M\left(\rho, \mu, C, \omega-\omega_{c}\right)\right)\right)^{-1} d \rho .
$$

An approximation to $r_{x_{0}}$ is

$$
r_{x_{0}}^{2}(x) \simeq r_{*}^{2}\left(1-e^{-\kappa x}+e^{\kappa\left(x_{0}-x\right)}\right)^{-1},
$$

where $\kappa=2 \mu \pi C^{-1}$. Due to the sign of the right-hand side of (3.6), $r_{x_{0}}$ is an increasing function of $x$. The phase $\varphi(x)$ satisfies

$$
\varphi(x)=\varphi_{0}+\omega x / C+G_{2}\left(x_{0}, x, \mu, \omega-\omega_{c}\right)
$$

with $G_{2}\left(x_{0}, x, \mu, \omega-\omega_{c}\right)=\int_{0}^{x} g_{2}\left(r_{x_{0}}^{2}(\chi), \mu, \omega-\omega_{c}\right) d \chi$. When $x \rightarrow \pm \infty$,

$$
G_{2}\left(x_{0}, x, \mu, \omega-\omega_{c}\right) / x \rightarrow g_{2}\left(r_{*}^{2} \quad \text { or } \quad 0, \mu, \omega-\omega_{c}\right)=O\left(\mu^{3 / 2}\right) .
$$

3.5. Solutions of (2.1). The small solutions to (2.1) are of the form (3.4), where $W$ and $\Lambda$ are responsible for the dependence in $\tau=\omega t$ and $z$. Substituting into (3.4) the solutions of (3.5) gives small solutions to (1.4)-(1.6). Approximations follow: indeed, the leading terms in the Taylor expansion of $\Lambda$ have been computed together with the first coefficients in the amplitude equation. Both families of bounded solutions to (3.5) correspond to time-periodic solutions to (2.1) in the form of travelling rolls and spacemodulated patterns.

3.5.1. Travelling Waves. With $r_{*}$ we get a family $R I(\omega, \mu, C)$ of travelling rolls, with wave number

$$
\omega C^{-1}+\ldots
$$

where "+..." is of the order of magnitude of $O\left(\left(r_{*}^{2}+\left(\omega-\omega_{c}\right)^{2}+|\mu|\right)\left(\omega-\omega_{c}\right)\right)+O\left(r_{*}^{2}+\right.$ $\left.O\left(r_{*}^{2}+\left(\omega-\omega_{c}\right)^{2}+|\mu|\right)^{2}\right)$. They are stationary in a frame moving at speed $C \vec{e}_{x}$ and correspond to the $P\left(\mu, k, X_{0}\right)$ of the subsection 1.3. A snapshot of the streamlines of an element of this family is represented in Figure 1. Our main interest is in the patterns with modulated amplitude given by (3.8). When the envelope is $r_{x_{0}}$, we obtain space modulated convective patterns $F I I\left(\omega, \mu, C, x_{0}\right)$. With $\varphi(x)=\omega x / C+\varphi_{0}+G_{2}(x)$, they correspond to

$$
\mathcal{U}=r_{x_{0}}(x) \sin (\pi z)\left(e^{i(\varphi(x)-\omega t)}\left(\begin{array}{c}
1 \\
i \pi \\
-i \\
\pi
\end{array}\right)+\text { c.c. }\right)+\Lambda\left(r_{x_{0}}(x) e^{i \varphi(x)}, r_{x_{0}} e^{-i \varphi}, \mu, \omega-\omega_{c}\right) .
$$

The corresponding fields of temperature and filtration speed oscillate with frequency $\omega$. 


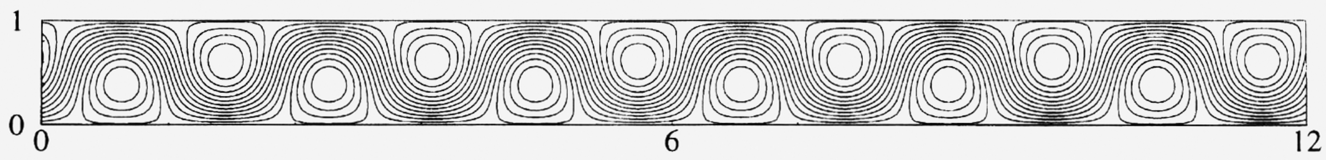

FIG. 1. Streamlines corresponding to travelling rolls $\omega-\omega_{c}=0.049$,

$\mathcal{R}=\mathcal{R}_{c}+0.1$ : analytical approximation

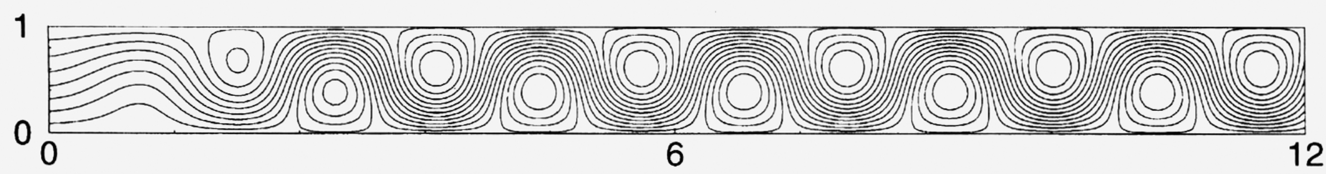

FIG. 2. Streamlines corresponding to front-like patterns with $\omega-$ $\omega_{c}=0.049, \mathcal{R}=\mathcal{R}_{c}+0.1$ : analytical approximation

For given $x_{0}$ the amplitude depends only on $x$ and increases monotonically in the streamwise direction; hence the patterns cannot be seen as being stationary from any uniformly moving frame. The wave-length is not exactly uniform: slight spatial modulations of the order of magnitude of $O\left(\mu^{3 / 2}\right)$ are observed.

The patterns are rolls whose amplitude is an increasing function of $x$, connecting flat isotherms and streamfunctions (upstream) to travelling rolls, analogous to the family $R I(\omega, \mu, C)$ downstream, as in Figure 2. The region connecting the conductive behaviour and the travelling rolls is linked to $x_{0}$ and stays fixed. Similar patterns have been observed in the numerical and experimental studies of [20] and [25] in fluid layers.

Our theoretical approach so far is unable to answer the question of whether some of these structures are physically relevant or not, with respect to their stability. We will see that a numerical simulation recovers some of the time-periodic solutions to (1.1)-(1.3): they are the ones that are stable in the conditions of the experiment.

4. Numerical results. Simulations of (1.1)-(1.3) in a long bounded rectangular subdomain $D=[0, \ell] \times[0,1]$ of the porous layer have been performed, with a finite volume method. Suitable boundary conditions at the inlet and at the outlet yield timeperiodic convective configurations that fit the patterns of Sec. 3.5.

4.1. Method. We solve (1.1)-(1.3) with a semi-implicit temporal integration, and the corresponding discretization is a second-order Euler's backward implicit scheme. At each time the spatial integration uses a finite volume method of the second order in space, on uniform staggered grids. We have tried several possibilities for the mesh, choosing the largest time and space steps such that smaller values do not change the computed time-frequency. We also have checked that the computational domain is long enough: larger values of $\ell$ do not change the frequency.

After stabilization, once time-periodic structures are reached, we determine their time frequency $\omega$, and compare with approximations to the $R I(\omega, \mu, C)$ and $F I I\left(\omega, \mu, C, x_{0}\right)$. Several boundary conditions have been tried at both extremities of $D$ : all of them have been found to induce near the outlet a perturbed zone which corresponds to one wavelength. More significant differences are observed near the inlet and in the bulk of the layer. 
4.2. Results. Enforcing space-periodic behaviours, we obtain travelling rolls: with $\ell=12$, the relative deviation from the corresponding $R I(\omega, \mu, C)$ is about $5 \%$, with respect to the $L^{2}([0,10] \times[0,1])$ norm, near the threshold. In the bulk of the layer the discrepancy is about $2 \%$. Enforcing inlet and outlet boundary conditions equal to analytical approximations to some $F I I\left(\omega, \mu, C, x_{0}\right)$ produces similar deviations (from the $\left.F I I\left(\omega, \mu, C, x_{0}\right)\right)$. The differences saturate at the value of the mesh which has been chosen according to Sec. 4.1: since we compare with truncated Taylor expansions (at low order) we cannot expect a very accurate fitting.

Other inlet conditions, which we think to be more natural and to resemble the conductive state also have been tried. They are $T(0, z, t)=1-z$ and $\vec{V}(0, z, t) \cdot \vec{e}_{x}=C$ : $\vec{V}(0, z, t) \cdot \vec{e}_{z}$ is free. With $\vec{V}(\ell, z, t) \cdot \vec{e}_{x}=C$ and $\partial_{x} T(\ell, z, t)=0$ at the outlet, we obtain front-like patterns, which are not very different from the $F I I$ in the bulk of the layer. They do not belong to this family, since the inlet conditions are incompatible with (3.9). Nevertheless the $L^{2}([0,10] \times[0,1])$ norm of the relative deviation from the corresponding $F I I\left(\omega, \mu, C, x_{0}\right)$ is about $7 \%$; in the middle of the layer it is less. We have plotted in Figure 3 the maximum of the vertical seeping velocity at various locations in the midplane, for different values of $\mu$ near the threshold with $C$ far away from zero: it fits the analytical amplitude (3.9) with $x_{0}=1.8$, with the exception of the very neighbourhood of the inlet. One checks in Figure 3 that the length of the simulated fronts is approximately proportional to $C \mu^{-1}$, near the threshold.

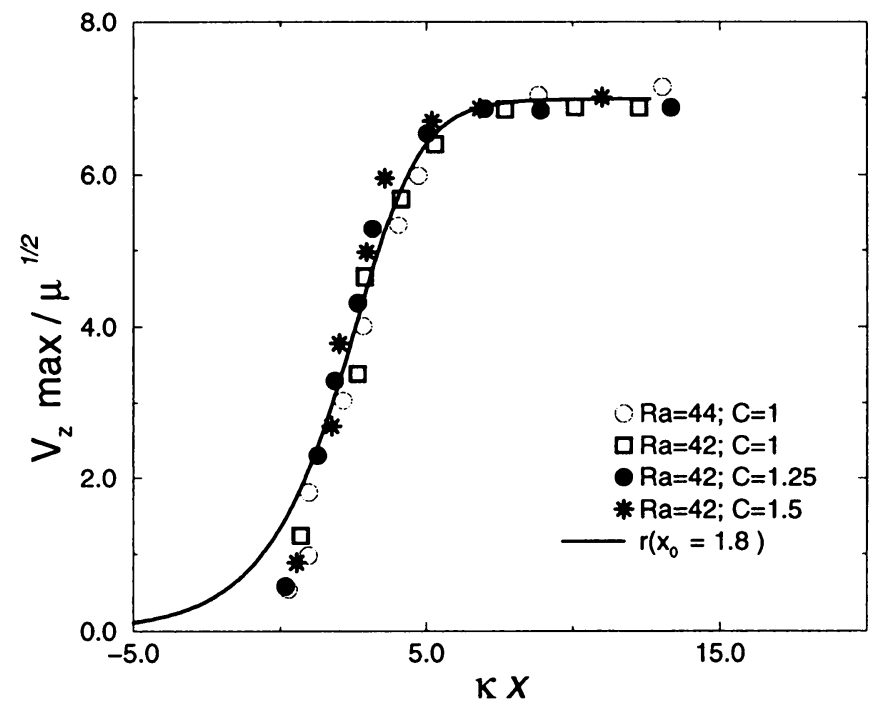

FIG. 3. Amplitudes of the simulated front-like patterns, compared with analytical expression

Conclusion. The center manifold method allows us to study bifurcations associated with the destabilization of a basic state, when some phenomenal parameter rises over its critical value. This approach had been used by [13] to study oscillatory fronts in fluid 
flows with or without the reflectional symmetry. With the help of this theory we have established that the system of partial differential equations, made of the Darcy law and the heat equation together with the equation of continuity, admits time-periodic solutions near the threshold. Convective structures, stationary with respect to a moving frame, solve the system: among them the quasi-periodic patterns and defect solutions (which cannot be durably seen as being different from periodic rolls by any observer in the laboratory frame) are not time-periodic, in contrast to the travelling waves. Front-like convective patterns, connecting flat isotherms (upstream to a fixed zone) to travelling waves, also exist and are time-periodic: they cannot be considered as being stationary in any moving frame with uniform speed. Oscillatory propagating fronts also had been shown by [5], [6], [8] and [9] to solve various parabolic equations with one spatial direction.

We have proved that the bifurcated travelling rolls and front-like structures exist. Up to now, we have no theoretical result about their stability. Indeed, our problem does not enter exactly the framework of the papers of [7], [10] and [24]: adaptations are at work. For this reason a numerical experiment has been implemented and some of these configurations have been simulated: they can be considered as being physically relevant under definite circumstances related with input and output boundary conditions. Indeed, this part of the study necessitated choosing the boundary conditions to be implemented at both extremities of $D$. Enforcing space-periodicity imposes the wave-length and therefore the dispersion relation (3.11) gives the frequency. Boundary conditions where this parameter does not appear explicitly were found to select the temporal period of the flow. It is clear that the inlet conditions are responsible for this, since changing the outlet conditions has no influence on the frequency. This fact had been put into evidence by the numerical work of [23], devoted to fluid layers.

The mathematical study of Sec. 3 is only relevant near the threshold, when the flowrate $C$ is kept away from a neighbourhood of zero, with an exception for the travelling waves which are stationary with respect to a moving frame and exist for all variations of $C$. The numerical method has been tested, by comparison with the analytical results. Parameters such as the size of the mesh have been optimized, and the method is ready to explore values of the filtration Rayleigh number far beyond the convective onset.

\section{Appendix.}

5.1. Proof of Lemma 2. For $f=\sum_{n \in \mathbb{Z}} f_{n} e^{i n \tau}$ and $g=\sum_{n \in \mathbb{Z}} g_{n} e^{i n \tau}$, let us look for $(\theta, \psi)$ of the form $\sum_{n \in \mathbb{Z}}\left(\theta_{n} e^{i n \tau}, \psi_{n} e^{i n \tau}\right)$. Since the real operator $-\partial_{z^{2}}^{2}$ with Dirichlet boundary conditions is bounded from below, the solution $\theta_{n}$ of

$$
\left(i n \omega+s^{2}\right) \theta_{n}-\partial_{z^{2}}^{2} \theta_{n}=f_{n}
$$

satisfies $\left\|\theta_{n}\right\|_{L^{2}[0,1]}\left(s^{2}+\pi^{2}\right)+n \omega\left\|\theta_{n}\right\|_{L^{2}[0,1]} \leq\left\|f_{n}\right\|_{L^{2}[0.1]}$. Consequently

$$
\left(\omega\|\theta\|_{\mathcal{H}^{1,0}}+\|\theta\|_{\mathcal{H}^{0,2}}+s^{2}\|\theta\|_{\mathcal{H}^{0,0}}\right) \leq 3\|f\|_{\mathcal{H}^{0,0}} .
$$

In view of $\omega^{2} n^{2}+s^{4} \geq 2 \omega n s^{2}$ we have $\|\theta\|_{\mathcal{H}^{1 / 2.0}} \leq(|s| \sqrt{2 \omega})^{-1}\|f\|_{\mathcal{H}^{0.0}}$. The scalar product of (5.1) with $\partial_{z^{2}}^{2} \theta_{n}$ yields $\left\|\partial_{z} \theta_{n}\right\|_{L^{2}[0,1]} \leq|s|^{-1}\left\|f_{n}\right\|_{L^{2}[0.1]}$; hence

$$
\|\theta\|_{\mathcal{H}^{0,1}} \leq|s|^{-1}\|f\|_{\mathcal{H}^{0,0}} .
$$


Thus, the unique solution to the first equation of $(2.5)$ in $\mathcal{H}_{0}^{0,1} \cap \mathcal{H}^{1,0}$ belongs to $\mathcal{H}^{2.0}$ and satisfies

$$
s^{2}\|\theta\|_{\mathcal{H}^{0.0}}+\omega|s|\|\theta\|_{\mathcal{H}^{1 / 2,0}}+|s|\|\theta\|_{\mathcal{H}^{0.1}}+\|\theta\|_{\mathcal{H}^{0.2}} \leq K_{1}(\omega)\|f\|_{\mathcal{H}^{0.0}} .
$$

Similarly, the second equation in (2.5) has one solution $\psi$ in $\mathcal{H}_{0}^{0.1} \cap \mathcal{H}_{0}^{1 / 2.1} \cap \mathcal{H}^{1 / 2.2}$, with

$$
s^{2}\|\psi\|_{\mathcal{H}^{1 / 2.0}}+|s|\|\psi\|_{\mathcal{H}^{1 / 2.1}}+\|\psi\|_{\mathcal{H}^{0.2}} \leq K_{1}(\omega)\|g\|_{\mathcal{H}^{1 / 2.0}}
$$

hence Lemma 2 is proved.

5.2. Proof of Lemmas 3 and 4.

Proof of Lemma 3. The problem (2.8) is equivalent to

$$
(\Theta, \Psi)=((i s+\alpha) \theta+h,(i s+\alpha) \psi+k)
$$

and

$$
\begin{aligned}
(\theta, \psi)+ & (i s+\alpha) R_{s}(C \theta+\mathcal{R} \psi, \mathcal{R} \theta)-\left(2 i \alpha s+\alpha^{2}\right) R_{s}(\theta, \psi) \\
& =R_{s}(f-C h+\mathcal{R} k+(i s+\alpha) h, g-\mathcal{R} h+(i s+\alpha) k)
\end{aligned}
$$

Let us take $s_{0}$ and $\alpha_{0}$ such that $\left|\alpha_{0} / s_{0}\right|, K_{1}(\omega)\left|s_{0}\right|^{-1}\left(1+\left|\alpha_{0} / s_{0}\right|\right)(\mathcal{R}+C)$ and $K_{1}(\omega)\left|s_{0}\right|^{-1}\left(\left|\alpha_{0}\right|+\left|\alpha_{0}^{2} / s_{0}\right|\right)$ are less than 1. By Neumann, series (5.4) has one solution $(\theta, \psi)$ in $\tilde{\mathcal{H}}_{s}$, with

$$
\begin{aligned}
\|(\theta, \psi)\|_{\tilde{\mathcal{H}}_{s}} \leq K^{\prime}\left(\omega, s_{0}, \mathcal{R}, C, \alpha_{0}\right) s^{-1} \| R_{s}(f-C h+\mathcal{R} k+ & (i s+\alpha) h, \\
& g-\mathcal{R} h+(i s+\alpha) k) \|_{\tilde{\mathcal{H}}_{s}}
\end{aligned}
$$

in view of (2.7). Hence Lemma 3 is proved.

Proof of Lemma 4. With $\left(L_{\mathcal{R} . C . \omega}-\lambda \mathrm{Id}\right) U=(0, f, 0,0),(2.10)$ is equivalent to $\left(L_{\mathcal{R} . C . \omega}-\lambda \mathrm{Id}\right) U^{\prime}=U$, which has a solution according to Lemma 3 .

Thus $\Theta^{\prime}-\lambda \theta^{\prime}=\theta, \Psi^{\prime}-\lambda \psi^{\prime}=\psi$ and

$$
\begin{aligned}
-\partial_{z^{2}}^{2} \theta^{\prime}+\omega \partial_{\tau} \theta^{\prime}-\lambda^{2} \theta^{\prime} & +C \lambda \theta^{\prime}-\mathcal{R} \lambda \psi^{\prime}=(2 \lambda-C) \theta+\mathcal{R} \psi \\
& -\partial_{z^{2}}^{2} \psi^{\prime}-\lambda^{2} \psi^{\prime}+\mathcal{R} \lambda \theta^{\prime}=2 \lambda \psi-\mathcal{R} \theta .
\end{aligned}
$$

The reasoning of the proof of Lemma 3 with $\lambda=\alpha+i s$ shows that

$\left\|\left(\theta^{\prime}, \psi^{\prime}\right)\right\|_{\tilde{\mathcal{H}}_{s}} \leq K^{\prime \prime}\left(\omega, s_{0}, \mathcal{R}, C, \alpha_{0}\right)\left(\left\|R_{s}((2 \lambda-C) \theta+\mathcal{R} \psi, 2 \lambda \psi)\right\|_{\tilde{\mathcal{H}}_{s}}+\mathcal{R}\left\|R_{s}(0, \theta)\right\|_{\tilde{\mathcal{H}}_{s}}\right)$.

However,

$$
\left\|R_{s}((2 \lambda-C) \theta+\mathcal{R} \psi, 2 \lambda \psi)\right\|_{\tilde{\mathcal{H}}_{s}} \leq K^{\prime \prime \prime}(\omega)(\mathcal{R}+2+C)\left(\|\theta\|_{\mathcal{H}^{0.0}}+\|\psi\|_{\mathcal{H}^{1 / 2.0}}\right)
$$

while $\left\|R_{s}(0, \theta)\right\|_{\tilde{\mathcal{H}}_{s}} \leq K^{\prime \prime \prime}(\omega)|s|^{-1}\|\theta\|_{\mathcal{H}^{1 / 2,0}}$. In view of Lemma 2 we have

$$
\left\|\left(\theta^{\prime}, \psi^{\prime}\right)\right\|_{\tilde{\mathcal{H}}_{s}} \leq K^{\prime \prime \prime \prime}\left(\omega, s_{0}, \mathcal{R}, C, \alpha_{0}\right) s^{-2}\|f\|_{\mathcal{H}^{0,0}}
$$

hence Lemma 4 is proved. 
5.3. Proof of Lemma 5. Expanding every $(h, f, k, g)$ in $\mathcal{H}$ by the Fourier series $\sum_{n \in \mathbb{Z}} e^{i n \tau}\left(h_{n}, f_{n}, k_{n}, g_{n}\right)$, let us search for solutions to $\left(L_{\mathcal{R}, C, \omega}-\lambda \operatorname{Id}\right) U=(h, f, k, g)$ of the form $\sum_{n \in \mathbb{Z}} e^{i n \tau} U_{n}=\sum_{n \in \mathbb{Z}} e^{i n \tau}\left(\theta_{n}, \Theta_{n}, \psi_{n}, \Psi_{n}\right)$. We have to solve

$$
\left(L_{\mathcal{R}, C, \omega}^{(n)}-\lambda \mathrm{Id}\right) U_{n}=\left(h_{n}, f_{n}, k_{n}, g_{n}\right)
$$

where the operator $L_{\mathcal{R}, C, \omega}^{(n)}$ is defined by

$$
L_{\mathcal{R}, C, \omega}^{(n)} U_{n} \equiv\left(\Theta_{n}, i n \omega \theta_{n}+C \Theta_{n}-\mathcal{R} \Psi_{n}-\partial_{z^{2}}^{2} \theta_{n}, \Psi_{n},-\partial_{z^{2}}^{2} \psi_{n}+\mathcal{R} \Theta_{n}\right) .
$$

The mapping $L_{\mathcal{R}, C, \omega}^{(n)}$ is continuous from $\hat{\mathcal{D}}=\left(\left(H^{2}[0,1] \cap H_{0}^{1}[0,1]\right) \times H_{0}^{1}[0,1]\right)^{2}$ to $\hat{\mathcal{H}}=$ $\left.\left(H_{0}^{1}[0,1]\right) \times L^{2}[0,1]\right)^{2}$, and the embedding $\hat{\mathcal{D}} \hookrightarrow \hat{\mathcal{H}}$ is compact. With $\rho_{\alpha_{0}, s_{0}} \equiv\{\lambda \in$ $\left.\mathbb{C} /|\operatorname{Re} \lambda| \leq \alpha_{0},|\operatorname{Im} \lambda| \leq s_{0}\right\}$ the following lemma just transposes to our situation arguments of [13].

LEMmA 6 . There exists $n_{0}$ such that for every $n \geq n_{0}$ and $\lambda$ in $\rho_{\alpha_{0}, s_{0}},\left(L_{\mathcal{R}, C, \omega}^{(n)}-\lambda \text { Id }\right)^{-1}$ is well defined in $\mathcal{L}(\hat{\mathcal{H}}, \hat{\mathcal{D}})$, with operator norm bounded by $K_{2}\left(\mathcal{R}, C, \omega, \alpha_{0}, s_{0}\right)$.

It is enough to prove the estimate for $\lambda=i s$, with real $s$, and then to apply the resolvent identity: observe that for every $f_{n}$ in $L^{2}[0,1]$ the problem

$$
(i s C+i n \omega) \theta_{n}-\partial_{z^{2}}^{2} \theta_{n}+s^{2} \theta_{n}=f_{n}
$$

has a solution $\theta_{n}=R_{C, s, n}^{\omega, \theta}\left(f_{n}\right)$ in $H^{2}[0,1] \cap H_{0}^{1}[0,1]$ with

$$
\left\|R_{C, s, n}^{\omega, \theta}\right\|_{\mathcal{L}\left(L^{2}[0,1], H_{0}^{1}[0,1]\right)} \leq\left((\omega n+s C)^{2}+s^{4}\right)^{-1 / 2}
$$

and $\left\|R_{C . s, n}^{\omega, \theta}\left(f_{n}\right)\right\|_{H^{2}[0,1]} \leq\left\|f_{n}\right\|_{L^{2}[0,1]}$.

The solution $\psi=R_{s}^{\psi}(g)$ to $-\partial_{z^{2}}^{2} \psi+s^{2} \psi=g$ in $[0,1]$ with $\psi(0)=\psi(1)=0$ satisfies

$$
\left\|R_{s}^{\psi}(g)\right\|_{H^{2}[0,1]} \leq\|g\|_{L^{2}[0,1]} .
$$

With this notation, (5.5) is equivalent to $\Theta_{n}=i s \theta_{n}+h_{n}, \Psi_{n}=i s \psi_{n}+k_{n}$ and

$$
\begin{gathered}
\left(\mathrm{Id}+\mathcal{R}^{2} s^{2} R_{C, s, n}^{\omega, \theta} R_{s}^{\psi}\right) \theta_{n}=R_{C, s, n}^{\omega, \theta}\left(f_{n}+\mathcal{R} k_{n}+i s h_{n}-C h_{n}+i s \mathcal{R} R_{s}^{\psi} g_{n}\right. \\
\left.-\mathcal{R} s^{2} R_{s}^{\psi} k_{n}+i s \mathcal{R}^{2} R_{s}^{\psi} h_{n}\right)
\end{gathered}
$$

which can be solved in $H_{0}^{1}[0,1]$ when $n \geq n_{0}$ with $\omega n_{0}>2 s_{0} C$ and $2 \mathcal{R}^{2} s_{0}^{2} \leq \sqrt{\omega n_{0}}$. One obtains

$$
\left\|\theta_{n}\right\|_{H_{0}^{1}[0,1]} \leq \hat{K}\left(\mathcal{R}, \omega, C, s_{0}\right) n^{-1 / 2}\left\|\left(f_{n}, g_{n}, h_{n}, k_{n}\right)\right\|_{\left(L^{2}[0,1]\right)^{4}}
$$

and then

$$
\left\|\theta_{n}\right\|_{H^{2}[0,1]}+\left\|\psi_{n}\right\|_{H^{2}[0,1]} \leq K^{\prime \prime \prime \prime}\left(\omega, C, s_{0}\right)\left\|\left(f_{n}, g_{n}, h_{n}, k_{n}\right)\right\|_{\left(L^{2}[0,1]\right)^{4}} .
$$

Therefore,

$$
\left\|\left(\theta_{n}, \Theta_{n}, \psi_{n}, \Psi_{n}\right)\right\|_{\hat{\mathcal{H}}} \leq K_{3}\left(\omega, C, s_{0}\right) n_{0}^{-1 / 2}\left\|\left(f_{n}, g_{n}, h_{n}, k_{n}\right)\right\|_{\hat{\mathcal{H}}} .
$$

By the resolvent identity, the above estimates yield for $\lambda$ in $\rho_{\alpha_{0}, s_{0}}$ :

$$
\left\|\left(L_{\mathcal{R}, C, \omega}^{(n)}-\lambda \mathrm{Id}\right)^{-1}\right\|_{\mathcal{L}(\hat{\mathcal{H}}, \hat{\mathcal{D}})} \leq K_{2}\left(\mathcal{R}, \omega, C, s_{0}, \alpha_{0}, n_{0}\right)
$$

and Lemma 6 is proved. 
Now we have to show that $\left(L_{\mathcal{R}, C, \omega}^{(n)}-\lambda \mathrm{Id}\right)^{-1}$ makes sense for $n \leq n_{0}$ except for a finite number of eigenvalues of the $L_{\mathcal{R} . C . \omega}^{(n)}$ in $\rho_{\alpha_{0}, s_{0}}$. We already know from Lemma 2 that $\left(L_{\mathcal{R}, C, \omega}-\lambda \mathrm{Id}\right)^{-1}$ and hence $\left(L_{\mathcal{R}, C, \omega}^{(n)}-\lambda \mathrm{Id}\right)^{-1}$ exist for $\lambda=i s$ when $s$ is real and large. Since the embedding $\hat{\mathcal{D}} \hookrightarrow \hat{\mathcal{H}}$ is compact, for each $n$ smaller than $n_{0}$ the resolvent $\left(L_{\mathcal{R}, C, \omega}^{(n)}-\lambda \mathrm{Id}\right)^{-1}$ exists except for a discrete set of $\lambda$ which are eigenvalues with finite multiplicities. In $\rho_{\alpha_{0}, s_{0}}$ with $n \leq n_{0}$ these $\lambda$ form a finite set.

Except for these $\lambda,\left(L_{\mathcal{R} . C . \omega}-\lambda \mathrm{Id}\right)^{-1}$ exists and is bounded in $S=\{\lambda \in \mathbb{C} /|\operatorname{Re}(\lambda)|<$ $\left.\alpha_{0}\right\}$ in view of Lemma 6 . These $\lambda$ are the eigenvalues of $L_{\mathcal{R}, C, \omega}$ in the strip $S$. In this subset of $\mathbb{C}$ the multiplicity of the eigenvalue $\lambda$ of $L_{\mathcal{R}, C, \omega}$ is less than the product by $n_{0}$ of the maximum of the multiplicities of $\lambda$ when it is considered as an eigenvalue of $L_{\mathcal{R}, C, \omega}^{(n)}$ with $n \leq n_{0}$. Hence Lemma 5 is proved.

\section{REFERENCES}

[1] R. A. Adams, Sobolev Spaces, Academic Press, 1975

[2] G. S. Beavers and E. M. Sparrow, Non-Darcy flow through fibrous media, Journal of Applied Mechanics 36, 711-714 (1969)

[3] J. L. Beck, Convection in a box of porous material saturated with fluid, Physics of Fluids 15, 1377-1383 (1972)

[4] H. R. Brand, R. J. Deissler, and G. Ahlers, Simple model for the Bénard instability with horizontal flow near threshold, Physical Review A 43, 4262-4268 (1991)

[5] P. Collet and J.-P. Eckmann, Instabilities and fronts in extended systems, Princeton Series in Physics, 1987

[6] P. Collet and J.-P. Eckmann, The existence of dendritic fronts, Comm. Math. Physics 107, 39-92 (1986)

[7] P. Collet, J.-P. Eckmann, H. Epstein, and J. Stubbe, A global attracting set for the KuramotoShivasinski equation, Comm. Math. Physics 153, 203-215 (1993)

[8] M. Combarnous, Convection Naturelle et Convection Mixte en Milieu Poreux, Thèse d'Etat, Paris, 1970

[9] J.-P. Eckmann and C. E. Wayne, Normal forms for parabolic partial differential equations, Ann. Inst. H. Poincaré Phys. Théor. 58, 287-308 (1993)

[10] J.-P. Eckmann and C. E. Wayne, The nonlinear stability of fronts for parabolic partial differential equations, Comm. Math. Physics 161, 323-334 (1994)

[11] J. Ettefagh, K. Vafai and S. Kim, Non Darcian effects in open ended cavities filled with a porous medium, J. of Heat Transfer 113, 747-756 (1991)

[12] G. Iooss and J. Los, Bifurcation of Spatially Quasi-Periodic Solutions in Hydrodynamic Stability Problems, Nonlinearity, vol. 3, 1990, pp. 851-871

[13] G. Iooss and A. Mielke, Bifurcating time-periodic solutions of Navier-Stokes equations in infinite cylinders, Journal of Nonlinear Science 1, 107-146 (1991)

[14] G. Iooss, A. Mielke, and Y. Demay, Theory of steady Ginzburg-Landau equations in hydrodynamic stability problems, European Journal of Mechanics B/Fluids 8, 229-268 (1989)

[15] G. Iooss and M.-C. Pérouème, Perturbed homoclinic solutions in reversible 1:1 resonance vector fields, Journal of Differential Equations 102, 62-88 (1993)

[16] R. M. Islam and K. Nandakumar, Multiple solutions for buoyancy induced flow in saturated porous media for large Peclet numbers, ASME Journal of Heat Transfer 108, 866-871 (1986)

[17] M. Le Cotillec, Instabilités dans une Couche Plane Poreuse Horizontale en Convection Mixte, Université de Paris VI, 1983

[18] J. L. Lions and E. Magenes, Problèmes aux Limites non Homogènes et Applications, vol. 1, edited by Dunod, 1968

[19] A. Mielke, Reduction of quasilinear elliptic equations in cylindrical domains with applications, Mathematical Methods in the Applied Sciences 10, 51-66 (1988)

[20] H. W. Müller, M. Lücke, and M. Kamps, Convective Patterns in Horizontal Flow, Europhysics Letters, vol. 10, no. 5,1989 , pp. $451-456$ 
[21] M.-C. Néel, Convection in a horizontal porous layer of infinite extent, European Journal of Mechanics B/Fluids 9, 155-176 (1990)

[22] X. Nicolas, A. Mojtabi, and J.-K. Platten, Two-dimensional analysis of the Poiseuille-Bénard flow in a rectangular channel heated from below, Physics of Fluids 9, 337-348 (1997)

[23] D. Roth, P. Büchel, M. Lücke, H. W. Müller, M. Kamps, and R. Schmitz, Influence of boundaries on pattern selection in throughflow, Physica D 97, 253-263 (1996)

[24] C. E. Wayne, Invariant manifolds for parabolic partial differential equations on unbounded domains, Arch. Rat. Mech. Anal. 158, 279-306 (1997)

[25] C. H. Yu, M. Y. Chang, and T. F. Lin, Structures of moving transverse and mixed rolls in mixed convection of air in a horizontal plane channel, Internat. J. of Heat and Mass Transfer 40, 333-346 (1997) 